\title{
A NONPOLYHEDRAL TRIANGULATED MÖBIUS STRIP
}

\author{
ULRICH BREHM
}

\begin{abstract}
We construct a triangulated Möbius strip with 9 vertices which is not embeddable into $\mathbf{R}^{3}$ such that all edges are straight line segments. It even cannot be immersed polyhedrally into $\mathbf{R}^{3}$.
\end{abstract}

For every integer $g \geqslant 1$ it is still an open problem whether there exists a triangulated closed orientable two-dimensional manifold of genus $g$ which is not embeddable into $\mathbf{R}^{3}$ such that all triangles are planar triangles with straight edges (cf. $[1,2])$. It is natural to ask the corresponding question for nonorientable two-dimensional manifolds with boundary. Theorems 1 and 2 (and the remark) answer that question completely also for immersions.

THEOREM 1. There exists a triangulated Möbius strip with 9 vertices which is not embeddable into $\mathbf{R}^{3}$ such that all edges are straight line segments.

Proof. (1) Let $I$ and $J$ be disjoint closed polygonal curves in $\mathbf{R}^{3}$ with three vertices. Then $|\operatorname{lk}(I, J)| \leqslant 1$ where $\operatorname{lk}(I, J)$ denotes the linking number of $I$ and $J$ (for the definition of linking number cf. [3]).

(2) The homotopy group of the Möbius strip is isomorphic to $\mathbf{Z}$. Let $\omega(J)$ denote the integer corresponding to the homotopy class of a closed curve $J$ on the Möbius strip. Let $I$ be the boundary curve of an embedded polyhedral Möbius strip in $\mathbf{R}^{3}$ and $J, J^{\prime}$ be simple closed polygonal curves in the relative interior of the Möbius strip, such that $|\omega(J)|=1$ and $\left|w\left(J^{\prime}\right)\right|=2$ (cf. Figure 1). Then $\operatorname{lk}(I, J)$ is an odd number (it is equal to the number of "twists" in the Möbius strip) and $\left|\operatorname{lk}\left(I, J^{\prime}\right)\right|=$ $2|\operatorname{lk}(I, J)|$, thus $\left|\mathrm{lk}\left(I, J^{\prime}\right)\right| \geqslant 2$.

(3) The triangulated Möbius strip given in Figure 2a cannot be embedded into $\mathbf{R}^{3}$ with straight edges, since for the triangular curves $I=1231$ and $J^{\prime}=4564$ we have $\left|\operatorname{lk}\left(I, J^{\prime}\right)\right| \geqslant 2$ by (2), (choose $J=7897$ ), which is impossible with straight edges by (1). In Figure $2 b$ we give another representation of the same triangulation (1 23 is not a triangle of the triangulation).

Figure 2a presents the Möbius strip as a rectangle with a pair of opposite edges identified with a twist and Figure $2 \mathrm{~b}$ presents it as a real projective plane with a triangle removed.

DEFINITION. Let us define a polyhedral immersion of a two-dimensional simplicial complex to be an immersion such that all triangles are planar and all edges are straight line segments.

Received by the editors November 17, 1982 and, in revised form, May 3, 1983.

1980 Mathematics Subject Classification. Primary 57Q35; Secondary 52A25, 57M20. 


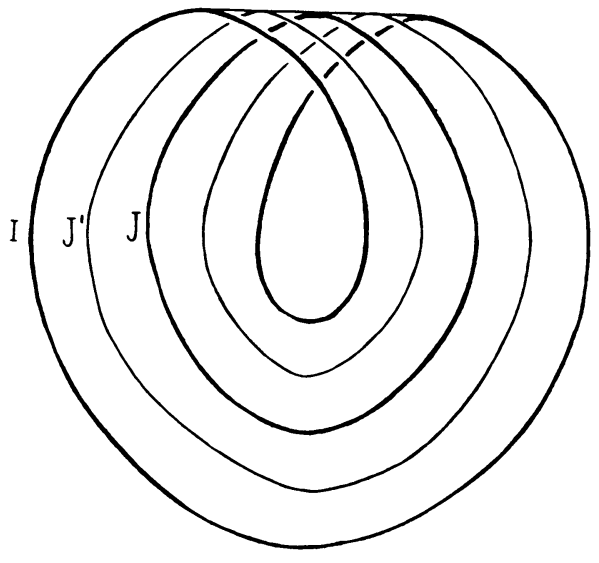

FIGURE 1

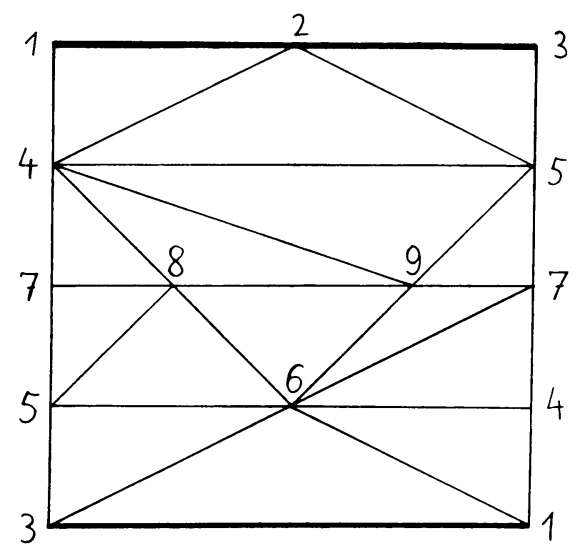

Figure 2a

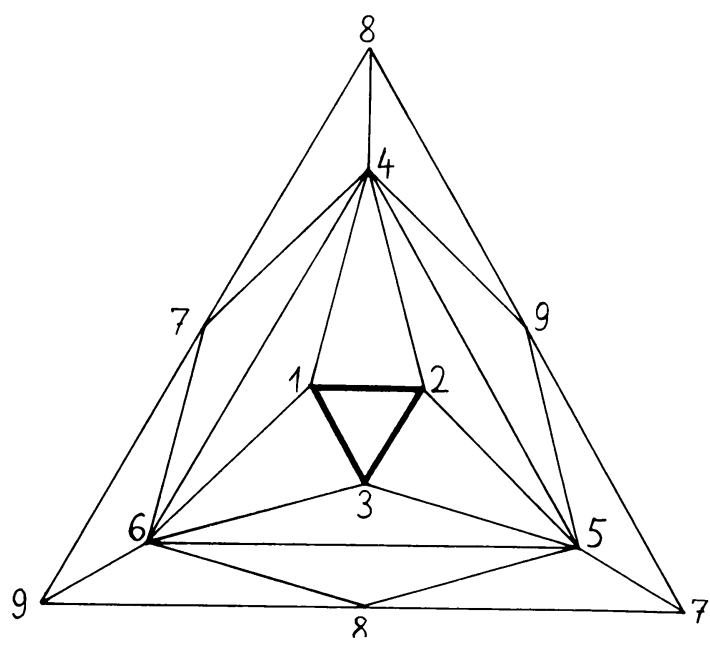

Figure 2b 
THEOREM 2. The triangulated Möbius strip given in Figure 2a cannot be polyhedrally immersed into $\mathbf{R}^{3}$.

Proof. (4) If a two-dimensional simplicial complex is polyhedrally immersed into $\mathbf{R}^{3}$ and if an edge $a_{1} a_{2}$ meets a triangle $b_{1} b_{2} b_{3}$ of the complex and if $a_{i} \neq b_{j}$ ( $i=1,2 ; j=1,2,3)$ then none of the triangles $a_{1} a_{2} b_{j}$ belongs to the complex.

(5) Let us assume that the Möbius strip given in Figure $2 a$ is polyhedrally immersed into $\mathbf{R}^{3}$. Let us define $M_{1}$ to be the compact Möbius strip which is contained in the given one and has boundary curve $J^{\prime}=4564$. (4) implies that an edge and a triangle of $M_{1}$ can only meet if they have a vertex in common. Consequently the immersion restricted to $M_{1}$ is already an embedding.

Now there exists also a neighborhood of $M_{1}$ in the given Möbius strip such that the immersion restricted to that neighborhood is an embedding. There exists a hexagonal curve $I_{1}$ (cf. Figure $3 \mathrm{a}$ or Figure $3 \mathrm{~b}$ ) in that neighborhood such that $I_{1}$ is disjoint to $M_{1}$ and homotopic to 4564 . So the immersion restricted to the compact Möbius strip which is contained in the given one and has $I_{1}$ as boundary curve is also an embedding.

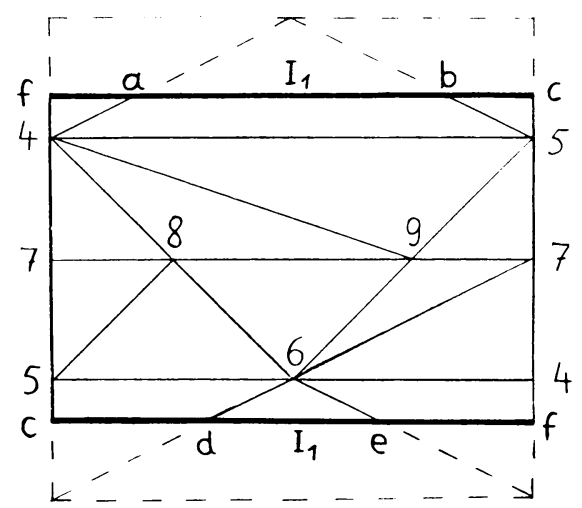

FIGURE 3a

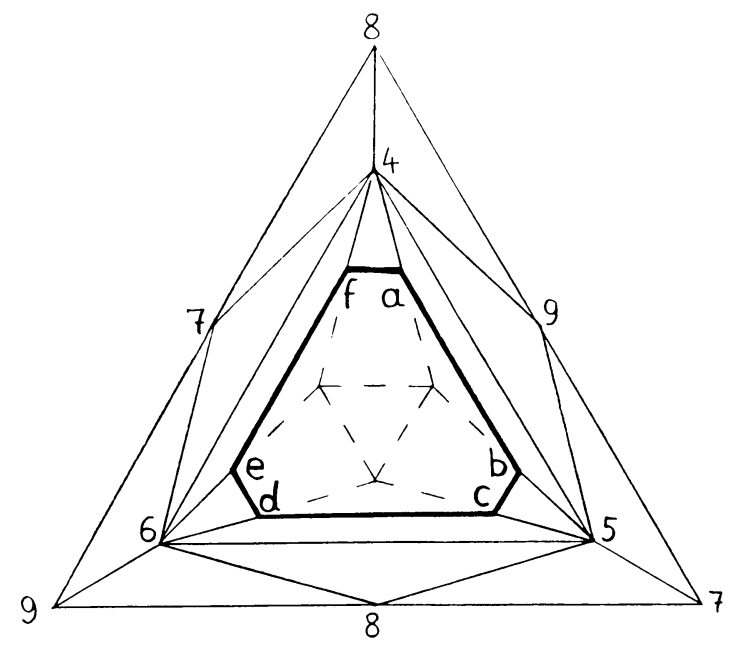

Figure 3b 
The edges $a b, c d$ and ef of $I_{1}$ (cf. Figure 3a or Figure $3 \mathrm{~b}$ ) cannot pierce the plane spanned by 4,5 and 6 . This implies $\operatorname{lk}\left(I_{1}, J^{\prime}\right) \leqslant 1$ in contradiction to (2).

REMARK. Since every two-dimensional nonorientable manifold $M$ with boundary contains a Möbius strip, we can triangulate $M$ such that it cannot be immersed (and consequently not be embedded) polyhedrally into $\mathbf{R}^{3}$.

\section{REFERENCES}

1. D. Barnette, Polyhedral maps on 2-manifolds, Convexity and Related Combinatorial Geometry (Proc. Second Conf. Univ. of Oklahoma) (D. C. Kay and M. Breen, eds.), Marcel Dekker, New York, 1982.

2. B. Grünbaum, Convex polytopes, Wiley, New York, 1967, p. 253.

3. D. Rolfsen, Knots and links, Math. Lecture Series 7, Publish or Perish, Berkeley, Calif., 1976.

Mathematisches Institut, Universität Freiburg, Albertstrasse 23B, D-7800 Freiburg, GERMANY 